\title{
Activity-Dependent Regulation of Inhibition via GAD67
}

\author{
C. Geoffrey Lau and Venkatesh N. Murthy \\ Department of Molecular and Cellular Biology and Center for Brain Science, Harvard University, Cambridge, Massachusetts 02138
}

Persistent alterations in network activity trigger compensatory changes in excitation and inhibition that restore neuronal firing rate to an optimal range. One example of such synaptic homeostasis is the downregulation of inhibitory transmission by chronic inactivity, in part through the reduction of vesicular transmitter content. The enzyme glutamic acid decarboxylase 67 (GAD67) is critical for GABA synthesis, but its involvement in homeostatic plasticity is unclear. We explored the role of GAD67 in activity-dependent synaptic plasticity using a mouse line $\left(\mathrm{Gad1}^{-1-}\right)$ in which GAD67 expression is disrupted by genomic insertion of the green fluorescent protein (GFP). Homozygous deletion of Gad1 significantly reduced miniature inhibitory postsynaptic current (mIPSC) amplitudes and GABA levels in cultured hippocampal neurons. The fractional block of mIPSC amplitude by a low affinity, competitive $\mathrm{GABA}_{\mathrm{A}}$ receptor antagonist was higher in GAD67-lacking neurons, suggesting that GABA concentration in the synaptic cleft is lower in knockout animals. Chronic suppression of activity by the application of tetrodotoxin (TTX) reduced mIPSC amplitudes and the levels of GAD67 and GABA. Moreover, TTX reduced GFP levels in interneurons, suggesting that GAD67 gene expression is a key regulatory target of activity. These in vitro experiments were corroborated by in vivo studies in which olfactory deprivation reduced mIPSC amplitudes and GFP levels in glomerular neurons in the olfactory bulb. Importantly, TTX-induced downregulation of mIPSC was attenuated in Gad $1^{-1-}$ neurons. Altogether, these findings indicate that activity-driven expression of GAD67 critically controls GABA synthesis and, thus, vesicular filling of the transmitter.

\section{Introduction}

Extreme and persistent alterations in network activity levels impair information propagation in neural circuits. In response to fluctuating activity levels, homeostatic mechanisms adjust excitatory and inhibitory transmission to maintain an appropriate degree of excitability (Davis and Bezprozvanny, 2001; Turrigiano and Nelson, 2004). In particular, chronic suppression of activity via pharmacological block of action potentials regulates inhibitory transmission by reducing the amplitude and frequency of miniature IPSCs (mIPSCs) in hippocampal neurons (Kilman et al., 2002; Rosato-Siri et al., 2002; Hartman et al., 2006). Although activity homeostatically regulates the quantal size of inhibition, the underlying mechanisms are unclear.

The inhibitory transmitter GABA is synthesized from glutamate by two distinct enzymes, glutamic acid decarboxylase (GAD) 67 (GAD67) and GAD65, with the cofactor pyridoxalphosphate (Soghomonian and Martin, 1998; Fenalti et al., 2007). Although both GAD67 and GAD65 contribute to GABA synthesis, their different localization, biochemistry, and knockout phenotypes suggest that they play differential roles in synaptic

Received March 13, 2012; revised May 2, 2012; accepted May 3, 2012.

Author contributions: C.G.L. and V.N.M. designed research;C.G.L. performed research; C.G.L. and V.N.M. analyzed data; C.G.L. and V.N.M. wrote the paper.

This study was supported by a National Institutes of Health Grant NS039059 (to V.N.M.). The GAD67-GFP mouse line was a kind gift from Yuchio Yanagawa, Gunma University, Maebashi, Japan.

The authors declare no competing financial interests.

Correspondence should be addressed to Dr. Venkatesh N. Murthy, Department of Molecular and Cellular Biology, Harvard University, Biological Laboratories, Room 4022, 16 Divinity Avenue, Cambridge, MA 02138. E-mail: vnmurthy@fas.harvard.edu.

DOI:10.1523/JNEUROSCI.1245-12.2012

Copyright $\odot 2012$ the authors $\quad 0270-6474 / 12 / 328521-11 \$ 15.00 / 0$ transmission and plasticity. GAD67 is found throughout the cell, whereas GAD65 preferentially localizes to synaptic terminals (Esclapez et al., 1994). Following its synthesis, GABA is loaded into vesicles by the vesicular GABA transporter (VGAT) and released from presynaptic terminals. As VGAT exhibits low affinity for GABA (McIntire et al., 1997), the cytosolic concentration of transmitter could be a critical determining factor of its vesicular loading (Mathews and Diamond, 2003; Hartman et al., 2006). Mice lacking GAD67 exhibit a 93\% reduction in cerebral GABA levels and die perinatally due to respiratory failure (Asada et al., 1997), suggesting an important contribution to proper neural function. In contrast, GAD65 knockout animals are largely normal in that their spontaneous IPSC (sIPSC) amplitudes are unaltered (Tian et al., 1999) and they live to adulthood albeit exhibiting susceptibility to seizures (Kash et al., 1997; Stork et al., 2000) and impaired visual cortical plasticity (Hensch et al., 1998). Various studies show that activity regulates the expression of both GAD isoforms and mIPSC amplitudes (Rutherford et al., 1997; Esclapez and Houser, 1999; Ramirez and Gutierrez, 2001; Hartman et al., 2006; Swanwick et al., 2006), but there is little evidence for a causal relationship between the two events. Since modulation of mIPSC amplitude by activity occurs, in part, via a change in the vesicular GABA content (Hartman et al., 2006), we hypothesized that GAD67 may play a causal role in linking activity to modulation of inhibition. Here, we investigated the role of GAD67 in controlling vesicular filling in basal transmission and synaptic homeostasis in vitro and in vivo. Using a combination of electrophysiology, immunofluorescent labeling, and genetics, we found that GAD67 critically regulates the cytosolic levels and vesicular filling of GABA and that this metabolic pathway is modulated in an activity-dependent manner. 


\section{Materials and Methods}

Primary neuronal cultures. The GAD67-GFP mouse was created previously by the knockin of EGFP into the exon 1 position of the Gadl gene, thereby abrogating expression of GAD67, the protein product of Gad1 (Tamamaki et al., 2003). GAD67-GFP mice were crossed to wild-type mice of Swiss Webster background. Mouse neonatal neurons [postnatal day (P)0], mice of either sex) from the hippocampus were treated with papain (Worthington), dissociated, and seeded on coverslips containing a rat glial feeder layer according to standard procedures (Hartman et al., 2006) and in accordance with institutional (Harvard University Institutional Animal Care and Use Committee, Cambridge MA) and national guidelines. Neuron/glia cocultures were grown in Neurobasal-A medium supplemented with B-27 and GlutaMax (both Invitrogen) without antibiotics to days in vitro (DIV) $12-16$ Gad1 $^{+1-}$ and ${ }^{-1-}$ animals were identified by the presence of GFP fluorescence in the intact brain, whereas $G a d 1^{-1-}$ mice were distinguished by the additional presence of cleft palate. Hence, $\mathrm{Gad} 1^{+1-}$ and $\mathrm{Gad} 1^{-1-}$ mice are genotypically +/GFP and GFP/GFP, respectively, but the former notation will be used throughout the manuscript to assess the effects of Gad1 deletion. All mouse genotypes were confirmed by PCR using the wild-type (WT) primer pair TR-1b (forward) 5'-GGCACAGCTCTCCCTTCTGTTT GC-3' and TR-3 (reverse) 5'-GCTCTCCTTTCGCGTTCCGACAG-3' (amplicon of $265 \mathrm{bp}$ ) and mutant primer pair TR-1b (forward) and TRGFP-8 (reverse) 5'-CTGCTTGTCGGCCATGATATAGACG-3' (amplicon of $564 \mathrm{bp}$ ). To globally suppress or elevate activity, $1 \mu \mathrm{M}$ tetrodotoxin (TTX, Tocris Bioscience) or $100 \mu \mathrm{M}$ picrotoxin (PTX, Tocris Bioscience) $/ \mathrm{KCl}(25 \mathrm{~mm})$, respectively, was added once to the cultures at DIV11-13, a period when synapses are deemed to be more mature. Two days following the addition of TTX or PTX, cells were used for electrophysiological recordings or fixed for immunofluorescent staining.

Electrophysiology. mIPSCs were recorded using the whole-cell patchclamp method in external solution containing (in $\mathrm{mm}$ ): $136 \mathrm{NaCl}, 2.5$ $\mathrm{KCl}, 10 \mathrm{HEPES}, 10$ D-glucose, $2 \mathrm{CaCl}_{2}, 1.3 \mathrm{MgCl}_{2}, \mathrm{pH} 7.4$, and 10 sucrose (osmolality 310-320 mOsm). Recording pipettes were pulled from borosilicate glass (WPI), and open-tip resistance was typically 3-5 M $\Omega$ with an internal solution of (in mM): $130 \mathrm{KCl}, 10$ HEPES, 0.2 EGTA, 10 DiTris-P-creatine, 2.5 glutamate, $3 \mathrm{MgATP}, 0.5 \mathrm{NaGTP}, \mathrm{pH} 7.3$ (osmolality 290-300 mOsm). To isolate mIPSCs, $500 \mathrm{~nm}$ TTX, $50 \mu \mathrm{m} 10$ D,L-APV (Tocris Bioscience) and $10 \mu \mathrm{M}$ CNQX (Tocris Bioscience) were added to the external solution $\left(V_{\mathrm{h}}=-70 \mathrm{mV}\right)$. Series resistance $(<25 \mathrm{M} \Omega)$ was regularly monitored during recordings, and cells were rejected if resistance changed by $>20 \%$ during the experiment. Data were filtered at 2 $\mathrm{kHz}$, digitized at $10 \mathrm{kHz}$, acquired through an Axopatch 200B amplifier (Molecular Devices) and custom-written scripts in Igor Pro 5 (Wavemetrics). mIPSCs were analyzed using the Mini Analysis 6 program (Synaptosoft) and OriginPro 7 (Microcal). The weighted decay time $\left(\tau_{\mathrm{w}}\right)$ of mIPSCs was calculated using a fit with two exponential time constants $(\tau 1$ and $\tau 2)$ and the formula $\tau_{\mathrm{w}}=\mathrm{I}_{1} /\left(\mathrm{I}_{1}+\mathrm{I}_{2}\right) \times \tau_{1}+\mathrm{I}_{2} /\left(\mathrm{I}_{1}+\mathrm{I}_{2}\right) \times \tau_{2}\left(R^{2}\right.$ $>0.9$ as accepting criterion). Statistical significance was evaluated by the two-tailed unpaired Student's $t$ test.

Immunofluorescent staining. Neurons were fixed in $4 \%$ formaldehyde for $10 \mathrm{~min}$ at room temperature (RT) and then blocked and permeabilized in $10 \%$ normal goat serum, $0.2 \%$ bovine serum albumin, and $0.1 \%$ Triton X-100 in PBS containing (in $\mathrm{mm}$ ): 10 phosphate, $138 \mathrm{NaCl}$, and $2.7 \mathrm{KCl}, \mathrm{pH}$ 7.4. Coverslips were incubated with primary antibodies (GAD65, 1:500, Hybridoma Bank; VGAT, 1:1000, Synaptic Systems; GAD67, 1:1000, Millipore Bioscience Research Reagents; GABA, 1:2000, Sigma; VGLUT1, 1:500, Synaptic Systems; $\mathrm{GABA}_{\mathrm{A}} \mathrm{R}$ subunit $\alpha 1,1: 100$, Alomone; $\mathrm{GABA}_{\mathrm{A}} \mathrm{R}$ subunit $\beta 2 / 3,1: 100$, Millipore Bioscience Research Reagents) overnight at $4^{\circ} \mathrm{C}$, washed and decorated with secondary antibodies conjugated to various Alexa Fluor fluorophores for $1 \mathrm{~h}$ at RT (1:1000, Invitrogen). Coverslips were mounted with anti-fade reagent Vectashield (Vector Laboratories) and imaged with confocal microscopy (Zeiss LSM510 or 700) using a $40 \times, 1.3$ numerical aperture oilimmersion objective at the Harvard Center for Biological Imaging (Cambridge, MA). Synaptic and somatic protein levels were analyzed using Image (NIH) or custom-written scripts in Matlab (MathWorks). Levels of GAD67, GFP, and GABA were quantified by measuring the mean intensity value in a hand-drawn selection of the soma. Levels of synaptic proteins (VGLUT1, VGAT, GAD65, GABA $\mathrm{R} \alpha 1$, and $\mathrm{GABA}_{\mathrm{A}} \mathrm{R} \beta 2 / 3$ ) were measured by placing small square boxes $(3 \times 3$ pixels $)$ over the labeled puncta within $100 \mu \mathrm{m}$ of soma. Fluorescence background (measured in adjacent nonpunctate neural regions) was subtracted for all puncta. Density was measured as the number of stained puncta per 20 $\mu \mathrm{m}$ length of dendrite. The $n$ reported is the number of puncta/somata analyzed (for intensity) or the number of dendrites (for density) from three or more independent cultures.

Hippocampal slice cultures. Coronal slices $(300 \mu \mathrm{m})$ of hippocampi from $\mathrm{Gad1} 1^{+/-}$animals (P5-7; mice of either sex) were prepared using a tissue slicer (Narishige) and cultured on a semiporous membrane (MilliCell, $0.4 \mu \mathrm{m}$ pore, $30 \mathrm{~mm}$ diameter; Millipore) for $7-10 \mathrm{~d}$ in minimal essential medium (MEM) supplemented with 25\% Earle's balanced salt solution, 23\% normal horse serum, $1 \mathrm{~mm}$ L-glutamine, and $36 \mathrm{~mm}$ [d]glucose. One-third of the culture medium was replaced every 3-4 d. To suppress activity, TTX $(1 \mu \mathrm{M})$ was added once at DIV5-7, and the organotypic slices were fixed after 3-5d, permeabilized, and stained for analysis as described above.

Deprivation of activity to the olfactory bulb in vivo. To suppress neural activity in the olfactory bulb (OB), we used the herbicide dichlobenil that, when injected intraperitoneally, selectively ablates olfactory sensory neurons in the epithelium (Yoon et al., 2005). WT (C57BL/6, 3 weeks old of either sex, for electrophysiology) or Gad1 ${ }^{+/-}$mice (7-8 weeks old of either sex, for immunostaining) were injected once with dichlobenil (100 $\mathrm{mg} / \mathrm{kg}$ ) or vehicle (DMSO, $1.7 \mu \mathrm{l} / \mathrm{g}$ body weight). For electrophysiological recordings, horizontal slices $(300 \mu \mathrm{m})$ of the $\mathrm{OB}$ were made with a vibratome (Leica) in ice-cold slicing solution containing (in $\mathrm{mM}$ ): 83 $\mathrm{NaCl}, 2.5 \mathrm{KCl}, 0.5 \mathrm{CaCl}_{2}, 3.3 \mathrm{MgSO}_{4}, 1 \mathrm{NaH}_{2} \mathrm{PO}_{4}, 26.2 \mathrm{NaHCO}_{3}, 22$ glucose, and 72 sucrose. Following a recovery period of $1-2 \mathrm{~h}$, slices were incubated in artificial CSF (ACSF) containing (in mM):119 NaCl, 2.5 $\mathrm{KCl}, 2.5 \mathrm{CaCl}_{2}, 1.3 \mathrm{MgSO}_{4}, 1 \mathrm{NaH}_{2} \mathrm{PO}_{4}, 26.2 \mathrm{NaHCO}_{3}$ and 22 glucose that is saturated with $95 \% \mathrm{O}_{2}$ and $5 \% \mathrm{CO}_{2}$ (carbogen). Recordings were obtained from external tufted (ET) cells using whole-cell patch-clamp technique, and these cells were distinguished from periglomerular (PG) cells by means of their input resistance (Tyler et al., 2007). Spontaneous IPSCs were recorded using a $\mathrm{Cs}^{+}$gluconate internal solution containing (in mM) $130 \mathrm{D}$-gluconic acid, $130 \mathrm{CsOH}, 5 \mathrm{NaCl}, 10 \mathrm{HEPES}, 12 \mathrm{Di}$-TrisP-creatine, 1 EGTA, 3 Mg-ATP and 0.2 Na-GTP (pH 7.3; 290 mOsm) at $V_{\mathrm{h}}=0 \mathrm{mV}$. For immunostaining, Gad1 ${ }^{+/-}$mice previously injected with dichlobenil or vehicle were anesthetized using ketamine $(100 \mathrm{mg} /$ $\mathrm{kg})$ and xylazine $(20 \mathrm{mg} / \mathrm{kg}$ ) and perfusion fixed transcardially with $4 \%$ formaldehyde. Sagittal OB sections $(70 \mu \mathrm{m})$ were incubated with antibody against GAD67 (1:2000), tyrosine hydroxylase (1:1000, Millipore), or olfactory marker protein $\left(1: 1000\right.$, Wako) overnight at $4^{\circ} \mathrm{C}$ and decorated with secondary antibody conjugated with Alexa Fluor 568 dye and mounted in VECTASHIELD (Vector Laboratories). Images were acquired with confocal microscopy (Zeiss) and analyzed using ImageJ.

\section{Results}

Gad 1 deletion reduces amplitude and frequency of mIPSCs

Since mice lacking GAD67, the gene product of Gad1, die perinatally (within hours after birth; Asada et al., 1997), we were unable to conduct studies in organotypic brain slices because it requires animals from P6 to P9 to preserve the synaptic connectivity and laminar architecture (Gogolla et al., 2006). Instead, we dissociated hippocampal neurons from newborn pups of WT, $\mathrm{Gad1}^{+/-}$(heterozygous), or Gad1 ${ }^{-/-}$(homozygous null) mice and seeded them on to a monolayer of astroglia. GFP was expressed in GABAergic interneurons in $\mathrm{Gad1}^{+/-}$and $\mathrm{Gad1} 1^{-/-}$ cultures and allowed their visual identification. To test how GABAergic transmission is affected by the deletion of Gad1, we performed whole-cell patch-clamp recordings from pyramidal neurons in WT, Gad1 ${ }^{+/-}$, and $\mathrm{Gad1}^{-1-}$ cultures (GFP-negative cells) and examined mIPSCs in the presence of TTX (500 nM), D,L-APV $(50 \mu \mathrm{M})$ and CNQX $(10 \mu \mathrm{M})$ at DIV12-16. The amplitude and frequency of mIPSCs of $\mathrm{Gad} 1^{+/-}$culture were similar 
a
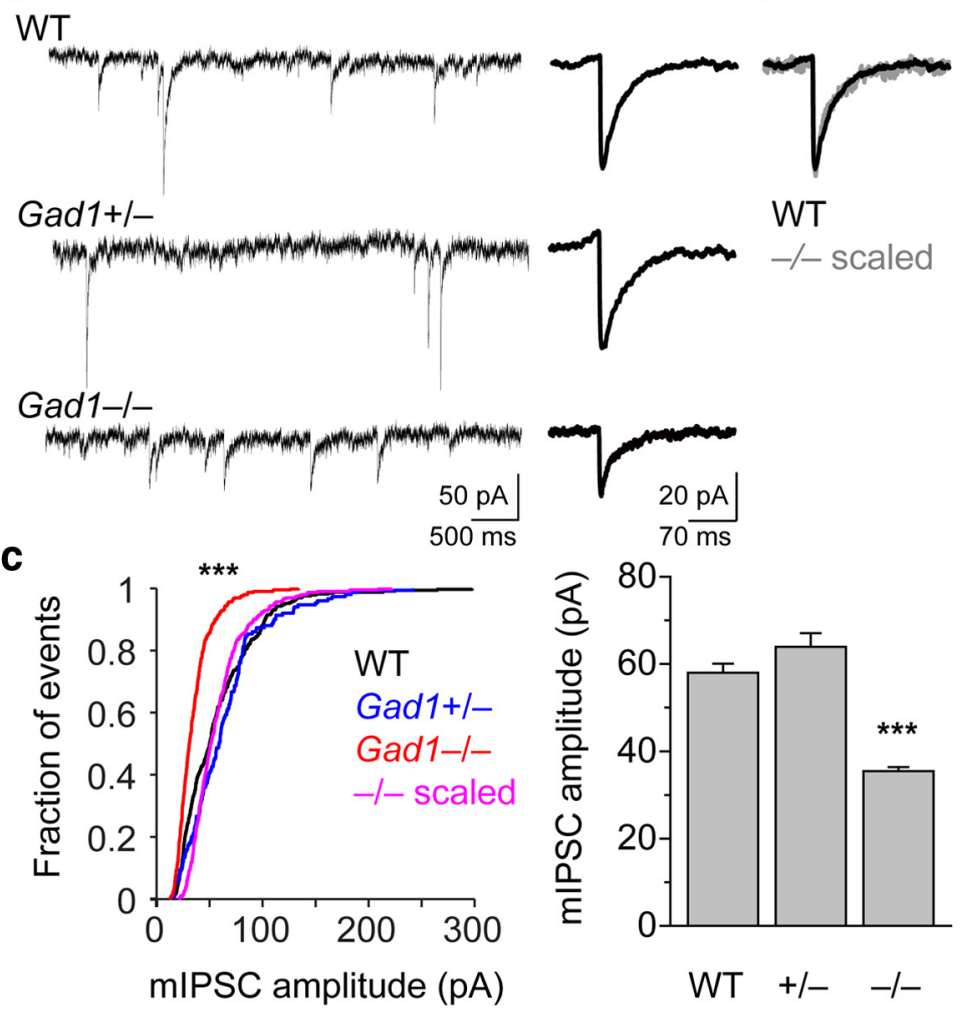

d

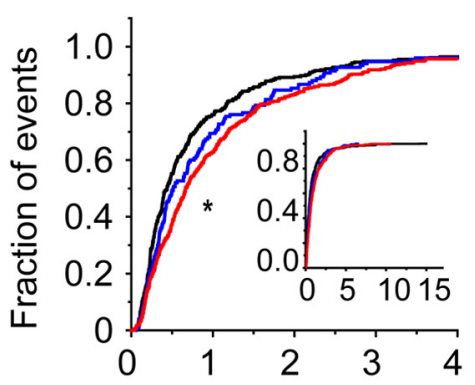

mIPSC interevent interval (s)

e

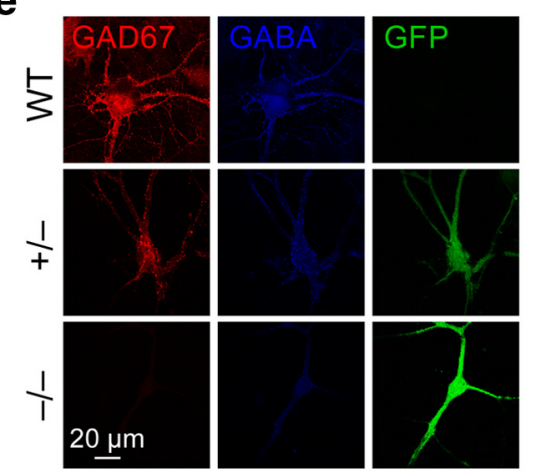

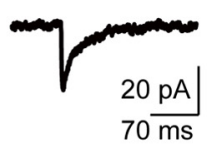

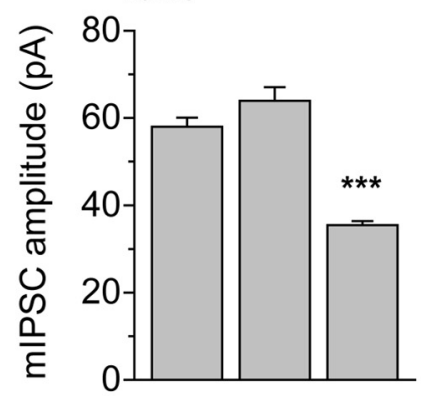

WT $+1--1-$

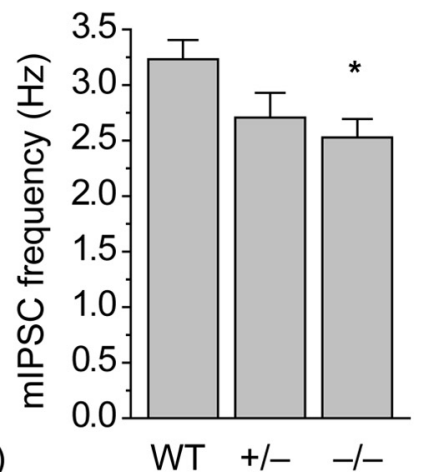

Figure 1. Gad1 deletion reduces mIPSC amplitude and frequency. $\boldsymbol{a}$, Left, Representative traces of mIPSCs recorded in the whole-cell patch-clamp mode (in $500 \mathrm{~nm}$ TTX, $50 \mu \mathrm{m}$ D, L-APV, and $10 \mu \mathrm{m}$ CNQX) using a KCl internal solution $\left(V_{\mathrm{h}}=-70 \mathrm{mV}\right.$ ) at DIV12-16. Gad1 ${ }^{-1-}$ neurons exhibited reduced mIPSC amplitude compared to WT or Gad1 ${ }^{+/-}$neurons. Right, Average traces of the different genotypes ( 30 consecutive mIPSCS). $\boldsymbol{b}$, Average trace from $\mathrm{Gad}^{-1-}$ neurons scaled up to match the amplitude of WT showed that the kinetics of mIPSCs were not altered. c, Cumulative distributions (left) and average amplitudes of mIPSCs (right) in WT, Gad $1^{+/-}$and $\mathrm{Gad}^{-1-}$ cultures. WT, $n=11$; Gad1 ${ }^{+/-}, n=5 ; \mathrm{Gad1}^{-1-}, n=11$. d, Cumulative distributions of the interevent interval (left) and average frequency of mIPSCS (right). WT, $n=11$; Gad1 ${ }^{+/-}, n=5 ; \mathrm{Gad}^{-1-}, n=11 . \boldsymbol{e}$, Left, Representative images from WT, Gad ${ }^{+/-}$, and $\mathrm{Gad}^{-1-}$ cultures immunofluorescently stained for GAD67 and GABA. Interneurons from $\mathrm{Gad}^{+/-}$and Gad ${ }^{-1-}$ cultures expressed GFP owing to the replacement of Gad 1 by GFP. Right, Average data of GABA

to those of WT cultures (WT vs Gad1 ${ }^{+/-}$ amplitude: $58.0 \pm 2.1 \mathrm{pA}$ vs $64.0 \pm 3.1$ $\mathrm{pA}, n=11$ vs $n=5, p>0.1$; WT vs Gad $^{+/-}$frequency: $3.23 \pm 0.17$ vs $2.71 \pm$ $0.22 \mathrm{~Hz}, n=11$ vs $n=5, p>0.07$; Fig. $1 a, c, d)$, suggesting that loss of one copy of Gad1 is not sufficient to alter inhibitory transmission. In contrast, deletion of both copies of Gad1 markedly reduced mIPSC amplitude (to $35.4 \pm 0.95 \mathrm{pA}, n=13, p<$ 0.0001 vs WT or Gad1 ${ }^{+/-}$; Fig. $1 c$ ) and frequency (to $2.53 \pm 0.17 \mathrm{~Hz}, n=13, p<$ 0.05 vs WT; Fig. $1 d$ ). When arithmetically scaled, the distribution of mIPSC amplitudes from $\mathrm{Gad} 1^{-1-}$ cultures resembled that of WT cultures (Fig. 1c). Since GABAergic mIPSCs could be detected even after loss of GAD67, it is likely that the remaining GABA is synthesized by GAD65. Loss of Gad1 did not affect the rise time (WT vs Gad1 ${ }^{-/-}, 1.56 \pm 0.18$ vs $1.97 \pm 0.22 \mathrm{~ms} ; p=0.18)$ or weighted decay times $\left(\tau_{\mathrm{W}}, \mathrm{WT}\right.$ vs $\mathrm{Gad} 1^{-1-}, 34.9 \pm$ 4.5 vs $32.3 \pm 2.8 \mathrm{~ms} ; p=0.63$ ) of mIPSCs (Fig. 1b). Passive membrane properties of Gad $1^{-/-}$neurons were not different from WT neurons in terms of capacitance (WT vs $\mathrm{Gad1}^{-1-}, 66.2 \pm 7.4$ vs $63.6 \pm 4.2 \mathrm{pF}$; $n=13$ vs $15, p=0.76$ ), input resistance $\left(\mathrm{WT}\right.$ vs $\mathrm{Gad} 1^{-1-}, 143.1 \pm 21.8$ vs $192.8 \pm$ $18.3 \mathrm{M} \Omega ; n=13$ vs $15, p=0.1$ ), or series resistance (WT vs Gad $1^{-/-}, 17.1 \pm 1.2$ vs $17.7 \pm 1.3 \mathrm{M} \Omega ; n=13$ vs $15, p=0.74)$. To assess whether Gadl deletion affects GABA synthesis, we next performed immunostaining for GAD67 and GABA. The lack of GAD67 staining in $\mathrm{Gad} 1^{-1-}$, but not WT or Gad1 ${ }^{+/-}$, cultures confirmed that GAD67 is indeed deleted in this genotype (Fig. 1e). Compared to interneurons in WT or $G a d 1^{+/-}$cultures, those in Gad1 $1^{-/-}$expressed significantly lower amounts of GABA in the cytosol (34 $\pm 6 \%$ of WT, $n=9, p<0.001$; Fig. $1 e$ ), suggesting that the reduction in quantal amplitude could be due to diminished GABA accumulation.

We next asked whether neuronal networks lacking GAD67 form and maintain glutamatergic and GABAergic synapses normally. Comparing WT and Gad1 ${ }^{-1-}$ neurons, we observed no difference in the density (per $20 \mu \mathrm{m}$ ) of excitatory or inhibitory synapses as revealed by immunofluorescent staining for the vesicular glutamate transporter VGLUT1 $(n=61$,

levels as shown on the left. Loss of both copies of Gad 1, but not one copy, reduced GABA levels. WT, $n=7 ; \operatorname{Gad1}^{+1-}, n=7$; Gad1 $^{-1-}, n=9$. ${ }^{*} p<0.05 ;{ }^{* * *} p<0.001$. All data are displayed as mean \pm SEM. a.u., Arbitrary units. 
a

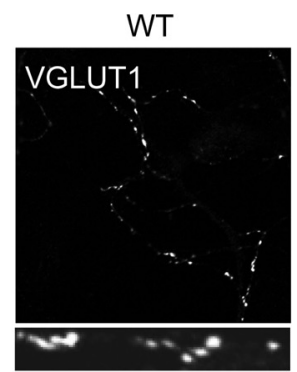

b

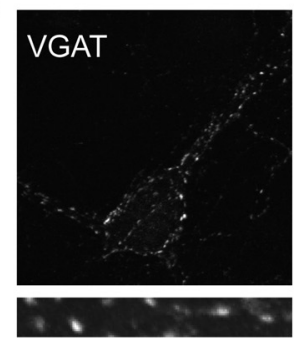

C

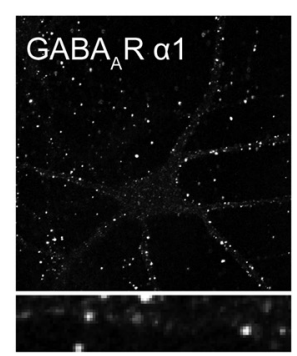

d

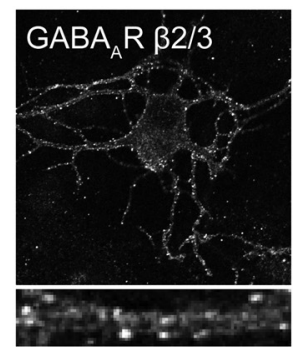

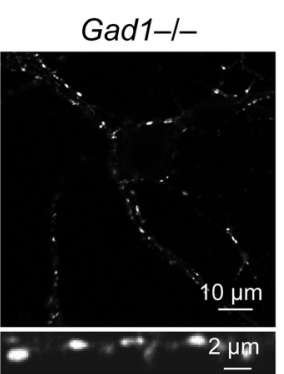
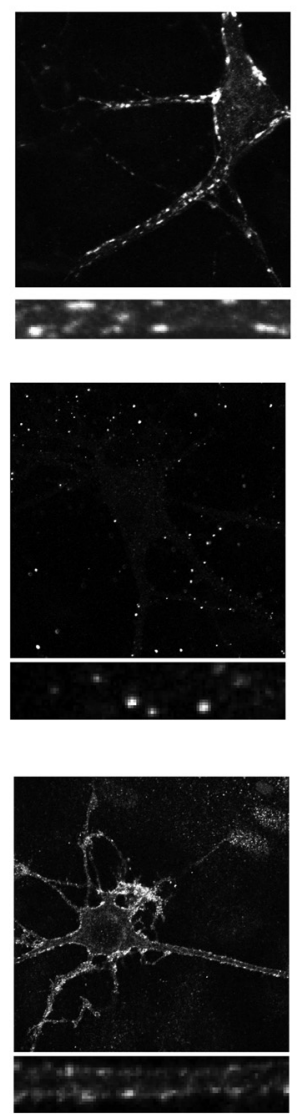
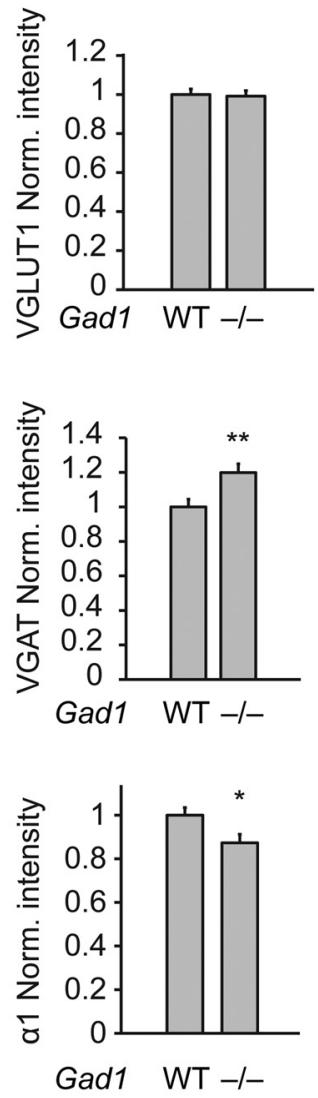

Gad1 WT -l-

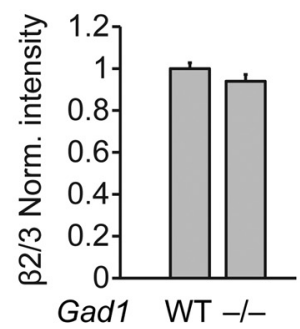

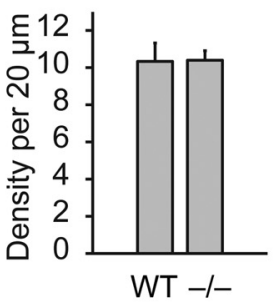
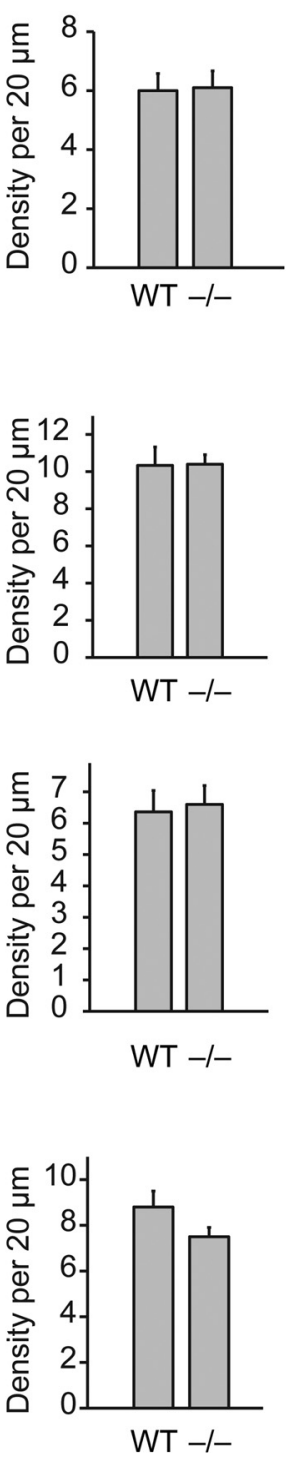

Figure 2. Normal development of excitatory and inhibitory synapses in Gad1 ${ }^{-1-}$ cultures. $\boldsymbol{a}$, Gad1 deletion did not alter the levels and density of the vesicular glutamate transporter VGLUT1, a marker for excitatory synapses $(n=61)$. $\boldsymbol{b}$, Gad 1 deletion did not alter the density of inhibitory synapse marker vesicular GABA transporter VGAT but increased its levels $(n=52)$. $\boldsymbol{c}, \boldsymbol{d}$, Gad 1 deletion minimally affected postsynaptic markers of GABA synapses $\mathrm{GABA}_{\mathrm{A}} \mathrm{R} \alpha 1(n=66)$ and $\beta 2 / 3(n=75)$ subunits. The densities of both subunits were unaltered, whereas levels of $G A B A_{A} R \alpha 1$ were slightly reduced (c). ${ }^{*} p<0.05 ;{ }^{* *} p<0.01$.

$p>0.9$, Fig. $2 a)$ or vesicular GABA transporter VGAT $(n=52$, $p>0.9$, Fig. $2 b$ ), respectively, of cultures at $12-16$ DIV. Interestingly, Gad1 deletion increased VGAT intensity by $20.0 \pm$ $5.0 \%(n=52, p<0.003$, Fig. $2 b)$, suggestive of compensation in vesicular uptake of GABA in the face of lowered cytosolic concentration. Moreover, Gad1 deletion minimally affected inhibitory postsynaptic receptors (reduced $\mathrm{GABA}_{\mathrm{A}} \mathrm{R}$ subunit $\alpha 1$ levels to $87.3 \pm 3.9 \%$ of WT, $n=66, p<0.05$, Fig. $2 c$; unaltered $\mathrm{GABA}_{\mathrm{A}} \mathrm{R}$ subunit $\beta 2 / 3$ intensity and density, $n=75, p>0.1$, Fig. $2 d$ ). These results suggest that GAD67 is not required for de novo formation of synapses.

Reduced vesicular GABA release from neurons lacking Gad1 We reasoned that if genetic ablation of $\mathrm{Gad} 1$ reduces synthesis of GABA and its vesicular filling, this will lead to lower GABA concentration in the synaptic cleft following quantal release. We used the low-affinity, competitive $\mathrm{GABA}_{\mathrm{A}}$ receptor antagonist (1,2,5,6-tetrahydropyridin-4-yl)methylphosphinic acid (TPMPA), to measure the relative concentration of GABA released quantally (Barberis et al., 2004; Liang et al., 2006). Application of TPMPA $(100 \mu \mathrm{M} ; 5 \mathrm{~min})$ reduced mIPSC amplitude by $18.4 \pm 0.5 \%$ in WT cultures as evidenced by the left shift in the cumulative probability distribution (Fig. $3 a, b$ ). The fractional block of mIPSC amplitude was significantly higher in $\mathrm{Gadl}^{-1-}$ cultures compared to WT (block of $27.0 \pm 0.4 \%$; WT vs Gad1 $1^{-1-}: n=180$ events from 6 cells vs 150 events from 5 cells, $p<0.0001$; Fig. $3 b$ ). To further examine the differential effects of TPMPA on WT and $\mathrm{Gad} 1^{-1-}$ cultures, we analyzed the relationship between the fractional TPMPA block and magnitude of mIPSC amplitude. For a wide range of amplitudes, mIPSCs in Gad1 ${ }^{-1-}$ neurons were more sensitive to TPMPA blockade relative to WT (Fig. 3c). Together, these findings demonstrate that the concentration of synaptically released GABA is lower in mice without GAD67. 
a
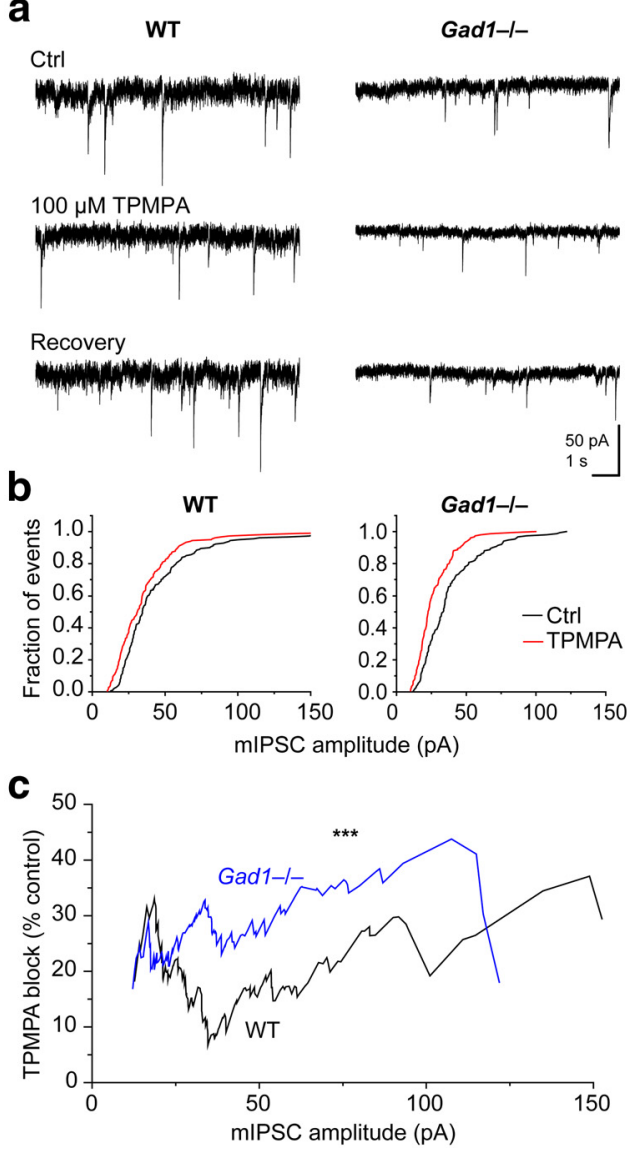

Figure 3. Gad1 deletion lowers vesicular filling of GABA. a, Application of the low-affinity, competitive GABA antagonist TPMPA reduced the amplitude of mIPSCs in WT (left) and $\mathrm{Gad1}^{-1-}$ (right) cultures. Washout of the drug allowed partial recovery of amplitudes. $\boldsymbol{b}$, Cumulative distribution of mIPSC amplitude in WT (left) and Gad ${ }^{-1-}$ (right) cultures. TPMPA (red line) induced a larger left shift in the distribution of mIPSC amplitude in Gad $1^{-1-}$ cultures compared to WT, suggesting lower vesicular content of GABA in cultures lacking GAD67.c, A plot of the degree of TPMPA block (as a percentage of baseline control) versus mIPSC amplitude revealed that mIPSCs are more sensitive to TPMPA in $\mathrm{Gad}^{-l-}$ for a large range of sizes. WT, $n=6$ cells; Gad1 ${ }^{-1-}, n=5$ cells; ${ }^{* * *} p<0.001$. Ctrl, Control.

\section{Inactivity reduces GAD67 and GABA levels}

Many studies have noted that pathological and physiological activity can regulate GAD67 mRNA and protein levels (Liang et al., 1996; Esclapez and Houser, 1999; Ramirez and Gutierrez, 2001; Patz et al., 2003), but it is unknown whether this modulation translates to altered synaptic function. To examine the relation between activity and GAD67 function, we dampened (by adding TTX) or elevated (with picrotoxin or $\mathrm{KCl}$ ) network activity for $2 \mathrm{~d}$ in WT, $\mathrm{Gadl}^{+/-}$, and $\mathrm{Gad1}^{-1-}$ cultures. In WT cultures, TTX significantly diminished GAD67 and GABA levels measured simultaneously in the same cells (GAD67 to $47.8 \pm 11 \%$ of control, $n=5, p<0.01$; GABA to $60 \pm 8 \%$ of control, $n=8, p<0.01$; Fig. $4 a, b)$. In contrast, chronic high activity with $\mathrm{KCl}(25 \mathrm{~mm}$ for $2 \mathrm{~d}$ ) significantly enhanced GAD67 (by $38.5 \pm 8.6 \%, n=11, p<$ 0.002 ) and GABA (by $62.2 \pm 16 \%, n=11, p<0.002$ ) levels (Fig. $4 a, b)$. TTX significantly reduced GAD67 and GABA expression in $\mathrm{Gad1}^{+/-}$cultures (GAD67 to $42.6 \pm 7.3 \%$ of control, $n=7$, $p<0.005$; GABA to $66.8 \pm 3.7 \%$ of control, $n=6, p<0.05$; Fig. $4 c, d)$. Conversely, PTX significantly augmented GABA levels with a tendency to increase GAD67 expression (GABA to $234 \pm$ $31 \%, n=5, p<0.01$; GAD67 to $123 \pm 18 \%, n=5, p>0.3$; Fig. $4 c, d)$. These results show that creating excess activity with PTX or
$\mathrm{KCl}$ increases GABA level, while reducing activity with TTX decreases it. For $\mathrm{Gad1}^{-1-}$ cultures, we did not perform immunostaining for GABA following TTX treatment due to the technical difficulty of quantifying a reduction in an already very low level of GABA. Interestingly, we found that inactivity strongly reduced the expression levels of GFP in interneurons of $\mathrm{Gad}^{-I}{ }^{-1}$ cultures (to $22.3 \pm 4.3 \%, n=10, p<0.0001$; Fig. $4 e, f$ ). Activity also regulated GFP levels in Gad1 ${ }^{+/-}$neurons, with TTX treatment depressing GFP fluorescence (to $56.5 \% \pm 9.1 \%, n=14, p<$ 0.001 ; Fig. $4 c, g$ ) and PTX enhancing it (to $149.0 \pm 23.4 \%, n=5$, $p<0.05$; Fig. $4 c, g$ ). In the GAD67-GFP mouse, egfp is inserted downstream of the endogenous Gad1 promoter, disrupting GAD67 expression. The TTX-induced loss of GFP expression suggests that activity directly modulates the Gad1 promoter in GABAergic interneurons, independent of any post-transcriptional regulation of GAD67. These results suggest that the biosynthetic pathway of GABA is dependent on GAD67 transcription and expression, which in turn is regulated by activity.

\section{Activity regulates GAD67 expression in hippocampal slice cultures}

The results we have described thus far indicate that activity regulates the production of GABA, in part, via expression of GAD67 in dissociated neurons. To test whether this effect occurs in a more intact preparation, we turned to organotypic cultures of hippocampal slices. Hippocampal slices (P5-7) from Gad1 ${ }^{+/-}$ animals maintained their overall circuit structure including the dentate gyrus, $\mathrm{CA} 3$, and CA1 areas when cultured in vitro (for 1-2 weeks; Fig. 5a). Application of TTX (1 $\mu \mathrm{M})$ for $2-5 \mathrm{~d}$ significantly reduced GFP (to $71.4 \pm 4.9 \%, n=28, p<0.01$; Fig. $5 b, c$ ) and GABA (to $73.2 \pm 7.0 \%, n=21, p<0.01$; Fig. $5 b, c$ ) levels in the $\mathrm{CA} 1$ area. These results corroborate our findings in dissociated culture (Fig. 4) and show that activity regulates the expression of GAD67 and GABA in slice cultures. Importantly, since GFP expression was driven by the endogenous Gad1 promoter, this regulation must engage, at least in part, a change in transcription.

\section{Impairment of synaptic homeostasis by Gad 1 deletion}

Our results implicate a molecular pathway involving GAD67 and GABA synthesis that is under the control of network activity. However, is GAD67 causally related to activity-dependent modulation of inhibition? To address this question, we determined whether synaptic homeostasis could be induced in $\mathrm{Gadl}^{-1-} \mathrm{cul}-$ tures with TTX and measured mIPSC amplitude and frequency. Application of TTX reduced the amplitude (control vs TTX: $58.4 \pm 3.8$ vs $33.7 \pm 2.9$ pA, $n=6, p<0.001$; Fig. $6 a, b)$ as well as frequency (control vs TTX: $2.71 \pm 0.19$ vs $2.05 \pm 0.19 \mathrm{~Hz}, n=6$, $p<0.05$; Fig. $6 a, b)$ of mIPSCs in WT cultures, consistent with observations in rat hippocampal neurons (Hartman et al., 2006). By contrast, in $\mathrm{Gad1} 1^{-1-}$ cultures TTX triggered a smaller fractional reduction in mIPSC amplitude (control vs TTX: $30.4 \pm 2.5$ vs $21.0 \pm 1.3 \mathrm{pA}, n=6, p<0.01$; Fig. $6 a, b)$ and prevented the change in frequency $(1.39 \pm 0.37$ vs $1.27 \pm 0.47 \mathrm{~Hz}, n=6, p>$ 0.5 ; Fig. $6 a, b$ ). TTX-induced fractional reduction in MIPSC amplitude in $\mathrm{Gadl}^{-l-}$ was $31.1 \pm 4.3 \%$ compared to $43.7 \pm 4.9 \%$ in WT $(n=6, p<0.001)$, in line with the notion that GAD67 is a target of activity-dependent regulation. In addition, TTX application downregulated the levels (to $53.3 \pm 3.2 \%, n=180$ synapses, $p<0.0001$; Fig. $6 c, d$ ) and density (from $8.3 \pm 0.7$ to $4.8 \pm$ 0.4 per $20 \mu \mathrm{m}, n=180$ synapses, $p<0.004$; Fig. $6 c, d$ ) of GAD65 at synaptic sites in WT cultures. In Gad1 ${ }^{-1-}$ cultures, homeostatic downregulation of GAD65 intensity was attenuated (to $76.8 \pm 2.7 \%, n=240$ synapses, $p<0.0001$; WT in TTX vs 
a

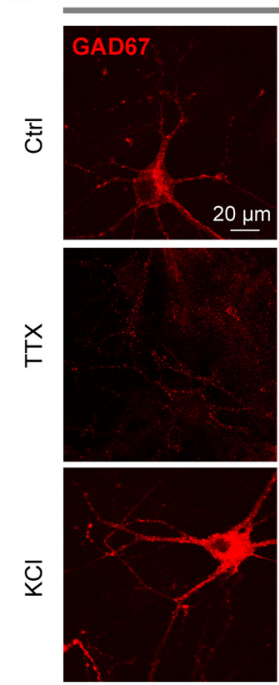

b

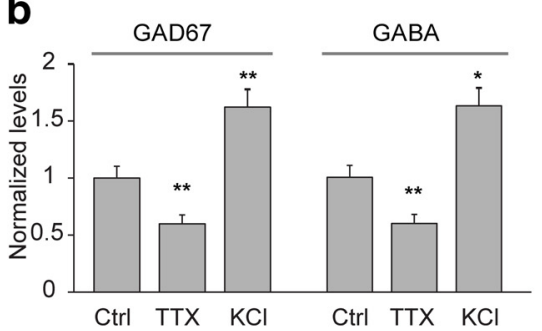

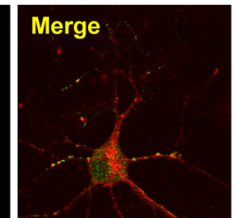
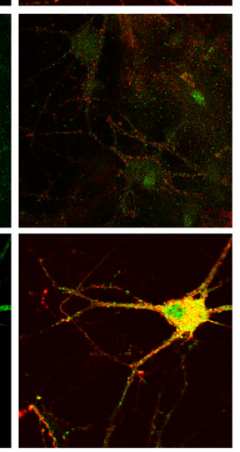

d
C

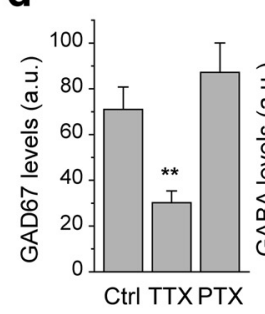

Gad1+l-

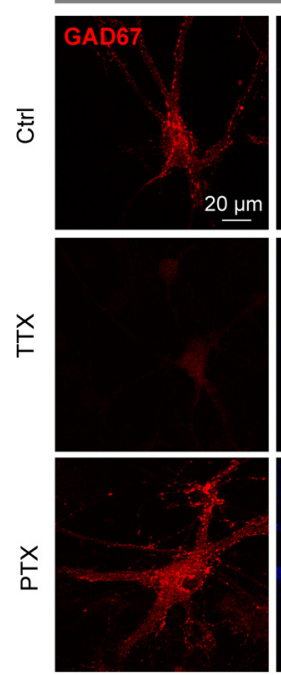

$20 \underline{\mu m}$
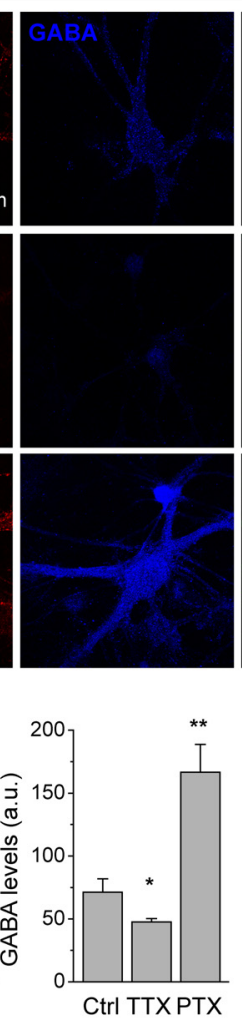

e
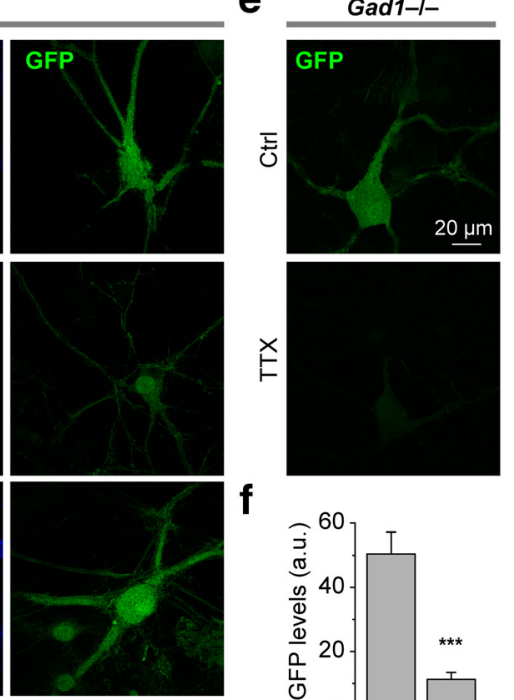

g
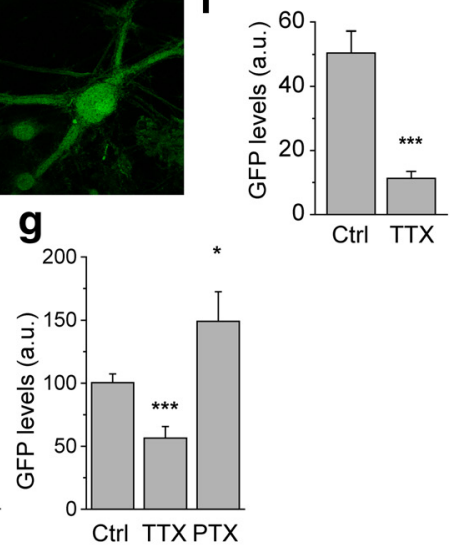

Figure 4. Activity regulates the levels of GAD67 and GABA. $\boldsymbol{a}$, Representative images showing that application of TTX (1 $\mu \mathrm{M})$ in WT cultures for $2 \mathrm{~d}$ reduced, whereas $\mathrm{KCl}(25 \mathrm{mM})$ increased, the intensity of GAD67 and GABA staining. $\boldsymbol{b}$, Average data for WT cultures $(n=8)$. $c$, Chronic inactivity (TTX) decreased whereas chronic hyperactivity (PTX) increased the levels of GAD67 and GABA in interneurons in Gad ${ }^{+/-}$culture. $d$, Average data for Gad ${ }^{+/-}$cultures. GAD67, $n=7 ; G A B A, n=6 . \boldsymbol{e}, \operatorname{In}$ Gad $1^{-1-}$ cultures, TTX reduced GFP expression in GABAergic interneurons even though GAD67 is absent. $\boldsymbol{f}$, Average for Gad ${ }^{-/-}$cultures; $n=10 . \boldsymbol{g}$, TTX $(n=14)$ decreased while PTX $(n=5)$ increased the expression of GFP in Gad $1^{+/-}{ }^{\text {neurons. }{ }^{*} p<0.05 ;{ }^{* *} p<0.01 ;{ }^{* * *} p<}$ 0.001 compared to control (Ctrl).

Gad1 $^{-1-}$ in TTX, $p<0.001$; Fig. $\left.6 c, d\right)$ whereas downregulation in density was abolished ( $\mathrm{Gad} 1^{-/-}{\mathrm{vs} \mathrm{Gad} 1^{-/-}}^{-}$in TTX $7.2 \pm 0.5$ vs $6.4 \pm 0.5$ per $20 \mu \mathrm{m}, n=6, p=0.3$; WT in TTX vs $G a d 1^{-1-}$ in TTX, $n=6, p<0.04)$.

Our results suggest an important role for GAD67 in the activity-induced modulation of synaptic inhibition. Since activity-dependent plasticity was not fully abolished in Gad1 ${ }^{-1-}$ cultures, additional molecular targets are likely to exist, including postsynaptic receptors (Kilman et al., 2002; Saliba et al., 2007; Rannals and Kapur, 2011).

\section{Activity regulates GAD67 expression and inhibitory transmission in vivo}

Based on the mechanisms we found in vitro thus far, can we infer that activity regulates GAD67 function in vivo? The mammalian olfactory bulb or $\mathrm{OB}$ is ideal for examining experience-induced alterations in synaptic transmission due to its direct connection to the sensory periphery and its amenability to activity manipulations. Olfactory sensory neurons release glutamate to activate principal neurons, the mitral and tufted cells (Wilson and Mainen, 2006; Fig. 7a). One class of principal cells called external tufted or ET cells is located in the glomerular layer and receives inhibition from interneurons called periglomerular or PG cells (Shao et al., 2009). At the first synapse in the $\mathrm{OB}$, sensory deprivation upregulates excitatory inputs to ET cells in a compensatory fashion (Tyler et al., 2007). We asked whether activity also alters inhibitory function, as a recent study suggests that sensory activity regulates GAD67 protein levels in PG cells (Parrish-Aungst et al., 2011).
To reduce sensory input to the glomerulus, we injected dichlobenil, a herbicide that ablates sensory neurons, intraperitoneally and compared its effects to vehicle control (DMSO). Dichlobenil ablated sensory neurons as evidenced by the loss of olfactory marker protein or OMP, a specific marker for sensory axons and terminals (Yoon et al., 2005; Fig. 7b). Dichlobenil reduced input activity to the glomerular layer since the levels of tyrosine hydroxylase or $\mathrm{TH}$, a protein commonly used to gauge activity in this system (Parrish-Aungst et al., 2011), were reduced (Fig. 7b). Activity deprivation by dichlobenil significantly reduced the amplitude of spontaneous IPSCs recorded from ET cells (from $35.9 \pm 1.5$ to $28.4 \pm 1.7 \mathrm{pA}, n=7, p<0.01$; Fig. $7 c, d$ ). We next examined the level of GAD67 protein in the glomerular region. Since it is difficult to decipher which inhibitory synapses are made on PG versus ET cell dendrites within the glomeruli with immunostaining, we examined GAD67-positive synapses on ET cell bodies, identified by the absence of GFP, in the juxtaglomerular region. Dichlobenil reduced the levels of synaptic GAD67 puncta surrounding ET cells (to $78.1 \pm 1.8 \%, n=210$, $p<0.0001$; Fig. $7 e, f$ ) as well as GFP in PG cells (to $80.1 \pm 1.7 \%$, $n=170, p<0.0001$; Fig. $7 e, f)$, consistent with a change in efficacy at inhibitory synapses. Overall, these results indicate that activity regulates inhibitory transmission and GAD67 expression at the earliest stage of olfactory processing in vivo.

\section{Discussion}

Alterations in network activity levels can induce compensatory changes in excitatory and inhibitory synaptic transmission that 
a
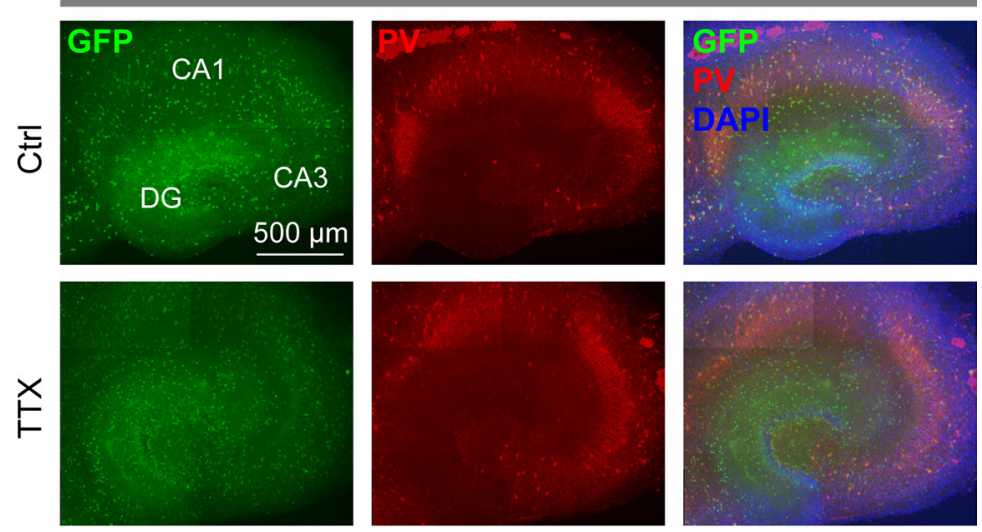

b
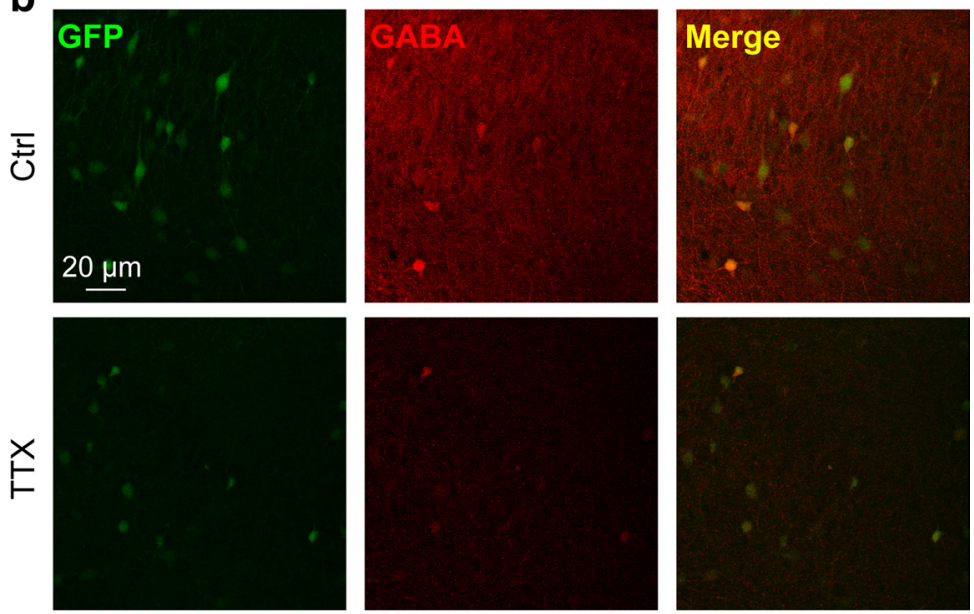

C

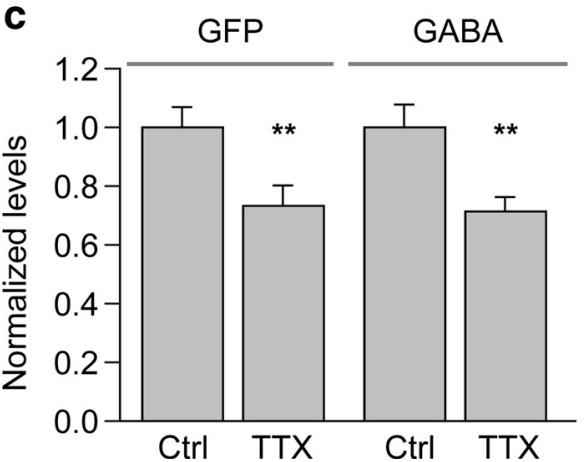

Figure 5. Activity regulates GAD67 and GABA expression in hippocampal slice cultures. $\boldsymbol{a}$, The laminar cytoarchitecture was maintained in cultures of GAD67-GFP heterozygous hippocampi. Dentate gyrus (DG), CA3, and CA1 areas can be visually distinguished. PV, Parvalbumin. $\boldsymbol{b}, \boldsymbol{c}$, Representative images $(\boldsymbol{b})$ and summary bar graphs $(\boldsymbol{c})$ showing that application of TTX (2-5 d) reduced the levels of GFP and GABA in the CA1 area. GFP, $n=28 ; \mathrm{GABA}, n=21$; ${ }^{* *} p<0.01$. Ctrl, Control.

Fourth, homeostatic regulation of mIPSC is blunted in $\mathrm{Gad1}^{-1-}$ cultures. These data converge on the notion that GAD67 expression regulates the levels of intracellular GABA, which in turn determines vesicular filling and synaptic strength.

A considerable body of evidence suggests that GAD67 expression is altered by activity (Esclapez and Houser, 1999; Ramirez and Gutierrez, 2001; Patz et al., 2003). In this study, we found that network activity positively regulates expression of GAD67 in hippocampal neurons in vitro and the $\mathrm{OB}$ in vivo. We showed that chronic inactivity reduces mIPSC amplitudes, GABA levels, expression of GAD67 protein, and a reporter gene driven by the Gad1 promoter. As GAD67 is the rate-limiting enzyme in GABA production (Chattopadhyaya et al., 2007; Huang et al., 2007), a simple scenario is that network activity activates Gad 1 transcription and GAD67 expression, thereby elevating GABA levels, which culminates in higher vesicular content. Homeostatic regulation of GAD67 expression and, thus, GABA levels, may represent a general mechanism to regulate excitability in hundreds of downstream neurons. We also made the surprising finding that activity modulates the gene expression of GFP in GABAergic interneurons. As GFP levels directly correlate with GABA levels in the GAD67-GFP mouse line (Suzuki and Bekkers, 2010), the amount of GFP expression can be used to gauge the overall activity level. Although GFP in this mouse line is commonly used as a marker for GABAergic neurons, one should be cautious because GFP expression is dynamically modulated by activity and therefore not constant. We propose that the GAD67-GFP culture system can be used as a method to screen various signaling pathways and small molecules that could influence GAD67 expression and inhibitory transmission. Modulation of neurotransmitter concentration via GAD67 may complement the well established method of regulating inhibitory

oppose the initial perturbation. Although recent studies show that chronic inactivity reduces mIPSC amplitudes, in part, by lowering vesicular content of GABA, the underlying molecular pathway is unknown. Here we identify GAD67 as a target of activity-dependent regulation that critically determines the cytosolic and vesicular concentration of GABA in interneurons. Several lines of evidence support this conclusion. First, Gad1 deletion reduces cytosolic GABA levels and mIPSC amplitude. The lower vesicular content of GABA is reflected by the higher fractional TPMPA block of quantal amplitudes in $\mathrm{Gad1}^{-/-}$neurons. Second, inactivity reduces GAD67 and GABA levels as well as mIPSC amplitude. Third, inactivity reduces the gene expression of Gad1, assessed using GFP as a reporter in vitro and in vivo. synaptic strength through changes in postsynaptic receptors (Kilman et al., 2002; Saliba et al., 2007; Rannals and Kapur, 2011).

What is the form of activity required to drive GAD67 expression? As silencing (by hyperpolarization) a single GABAergic interneuron does not alter its GAD65 expression (Hartman et al., 2006), it is probable that some soluble factor, regulated by network activity, determines GAD65 expression. Similarly, it is possible that network activity, via soluble paracrine signals such as brain-derived neurotrophic factor (BDNF) (Rutherford et al., 1997) or TNF- $\alpha$ (Stellwagen and Malenka, 2006), regulates GAD67 expression. Regulation of Gad1 promoter by activitydependent transcription factors such as Krox-24 (also known as Zif268 or Egr1) (Yanagawa et al., 1997), histone acetylation 
a
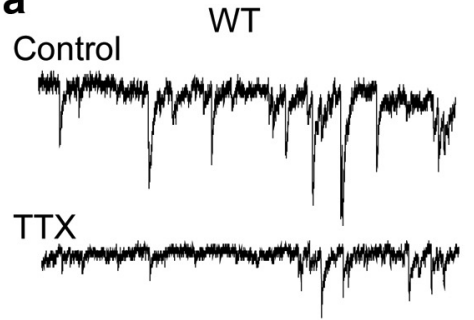

b

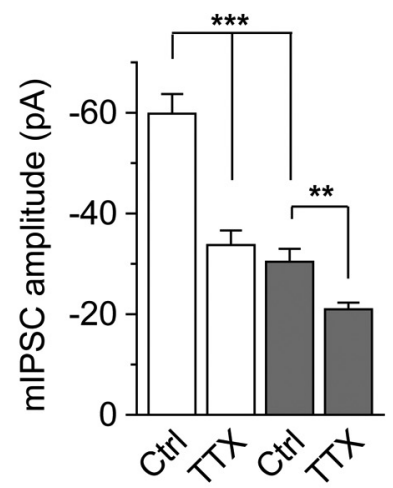

C
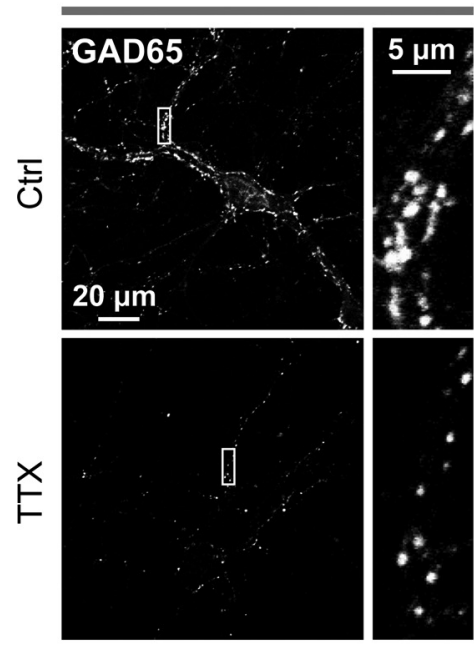

d

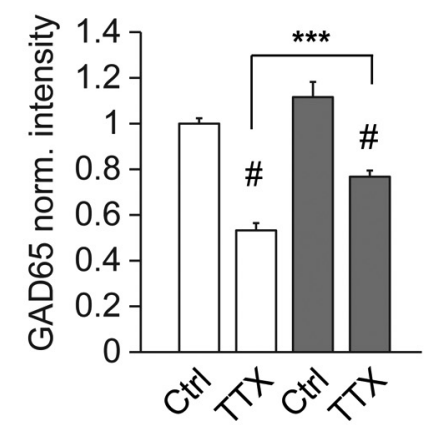

Gad1-I-
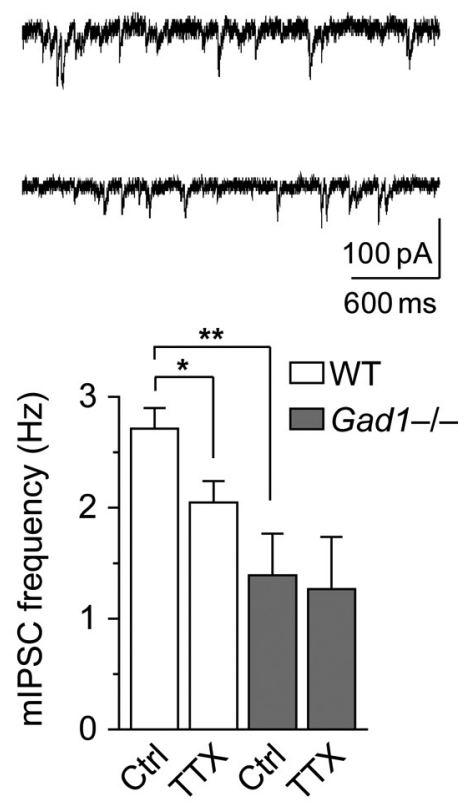

Gad1-/-
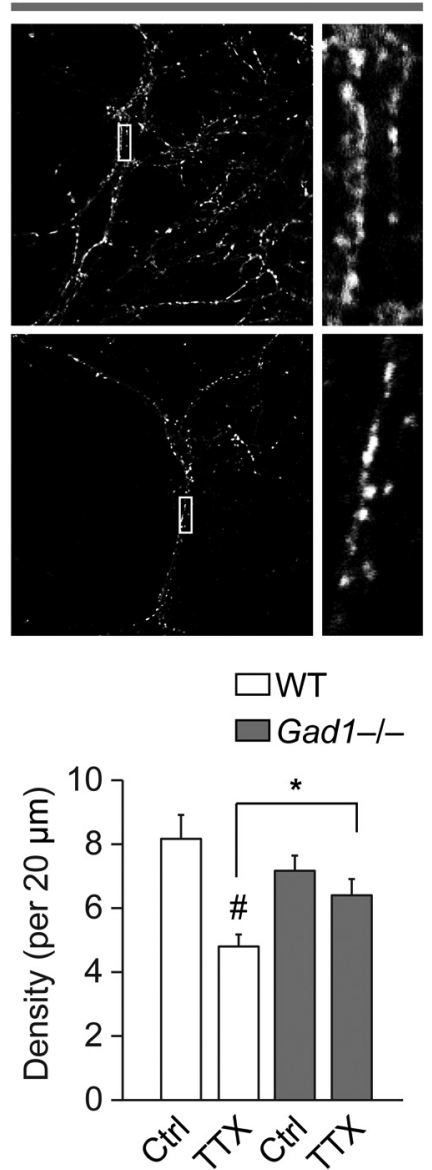

Figure 6. Impaired activity-dependent downregulation of mIPSCs and GAD65 in Gad $1^{-1-}$ neurons. $\boldsymbol{a}$, Representative traces showing that chronic application of TTX in WT cultures reduced mIPSC amplitude and frequency. Gad1 deletion attenuated the TTX-induced downregulation of mIPSC amplitude and prevented the change in frequency. $\boldsymbol{b}$, Summary of all data $(n=6)$. $\boldsymbol{c}$, Representative images (low and high magnifications) showing the regulation of GAD65 expression by TTX in WT and Gad $1^{-1-}$ cultures. The intensity level of GAD65 in Gad1 ${ }^{-1-}$ was similar to that of WT ( $p>0.08$ ). While TTX reduced GAD65 levels in WT cultures, this downregulation was attenuated in Gad $1^{-1-}$ neurons. In contrast, TTX-induced downregulation of GAD65 density is

(Tremolizzo et al., 2002), and DNA methylation (Kundakovic et al., 2007) can be some of the ways by which activity promotes GAD67 expression. In the OB, GABAergic PG cells do not always receive direct inputs from sensory neurons (Shao et al., 2009). Deprivation of activity may indirectly reduce excitatory input on to PG cells via a suppression of ET cell activity, enforcing a gain control mechanism. Examination of GAD67 expression control using molecular tools such as RNA interference and optogenetics will provide better insights into the mechanisms behind activity-dependent homeostasis.

In the cerebellum, homozygous deletion of $\mathrm{Gad} 1$ reduces mIPSC amplitude by $>70 \%$ and GABA levels by as much as 84\% (Obata et al., 2008). In this study, Gad 1 deletion reduces mIPSC by $39 \%$ and GABA levels by $58 \%$ in hippocampal neurons. The fact that we do not observe the same drastic impairment in GABAergic function may be due to the more redundant nature of GAD65 in the hippocampus and highlights a region-specific usage of the two GAD isoforms. While GAD65 is not involved in determining IPSC amplitude (Tian et al., 1999; Kubo et al., 2009), it is critical for maintaining tonic inhibition (Kubo et al., 2009; Song et al., 2011). It would be interesting to test, in a slice or in vivo model, the role of GAD67 on tonic inhibition. A myriad of compensatory responses may occur in $\mathrm{Gad1}^{-1-}$ neurons, complicating the effects during homeostatic plasticity. However, our data suggest that these effects are minor, as there were minimal changes in postsynaptic $\mathrm{GABA}_{\mathrm{A}}$ receptors (Fig. 2) and no change in GAD65 levels or density (Fig. 6), mIPSC kinetics (Fig. 1), cell size, or resistance. Gad1 deletion affects mIPSC amplitude to a greater degree than frequency, but this could be due to small mIPSCs falling below detection threshold. Interestingly, Gadl deletion increases synaptic VGAT expression, similar to the oppositely regulated levels of GAD65 and VGAT by BDNF (Henneberger et al., 2005). Therefore, individual synapses may attempt to fill vesicles to some desired yet unknown level. Despite these compensatory responses, the degree of

$\leftarrow$

completely abolished in $\mathrm{Gad1}^{-1-}$ neurons compared to WT. The effect of Gad1 deletion on GAD65 puncta intensity and density mirrors that of mIPSC amplitude and frequency, respectively. $\boldsymbol{d}$, Summary of the analysis of GAD65 intensities. WT, $n=180$ synapses; Gad1 ${ }^{-1-}, n=240$ synapses. ${ }^{*} p<$ $0.05 ;{ }^{* *} p<0.01 ;{ }^{* * *} p<0.001 ;{ }^{*} p<0.001$ versus control (Ctrl; no TTX). norm., Normalized. 
a

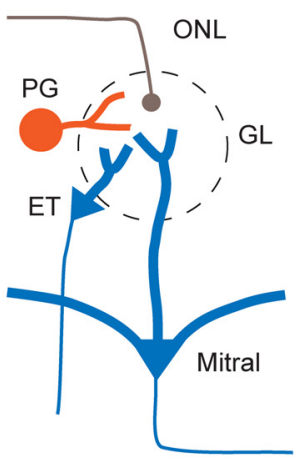

b

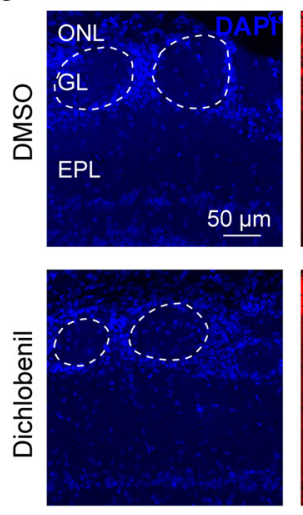

e
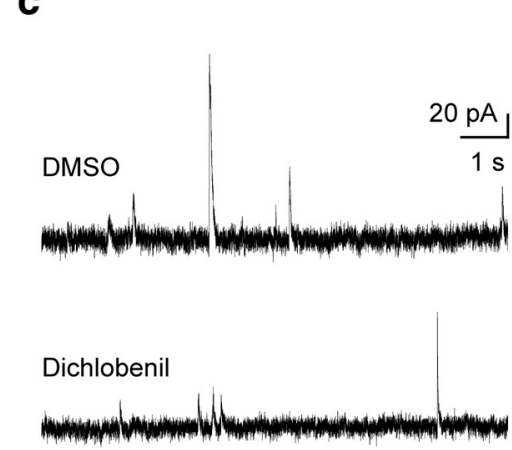

d

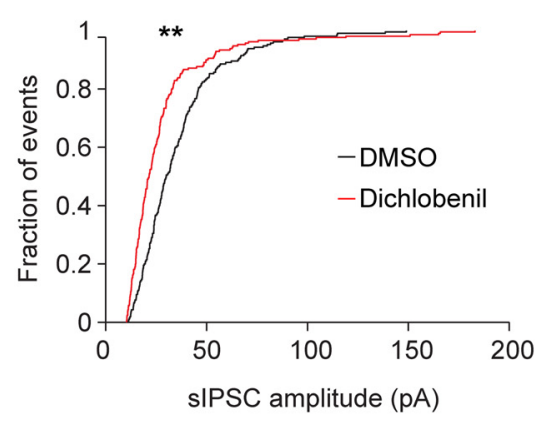

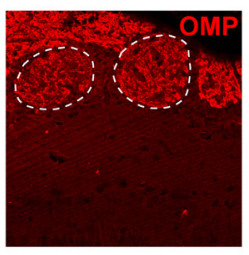
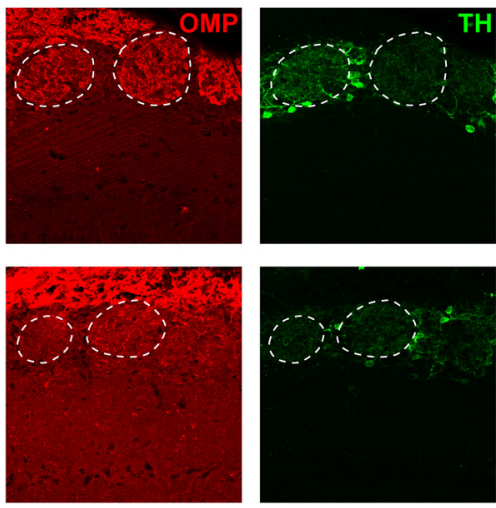

Gad1+/-
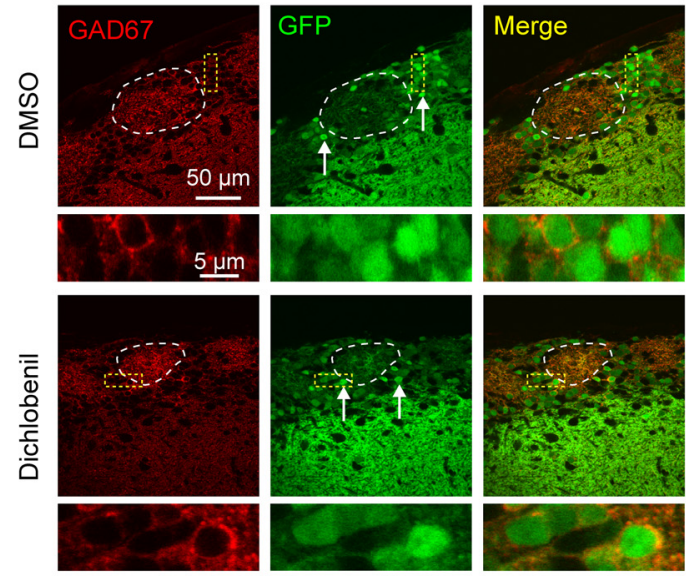

f

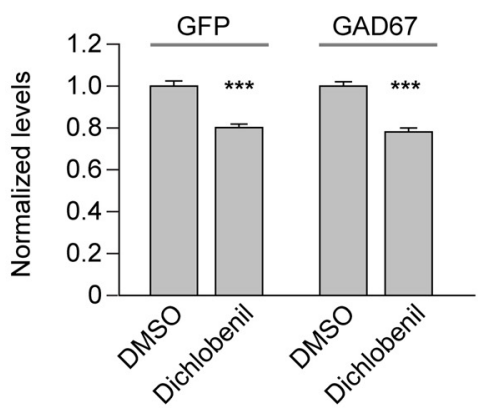

Figure 7. Experience regulates inhibitory transmission and GAD67 expression in the OB in vivo. $a$, Schematic diagram of the glomerular circuit in the OB. Olfactory sensory neurons (OSNs) release glutamate to activate ET and mitral cells. PG cells release GABA and modulate synaptic transmission in the glomerular layer (GL). $\boldsymbol{b}$, Seven to ten days after injection of dichlobenil to ablate OSNs and reduce odor-evoked activity, olfactory marker protein (OMP) staining was largely absent in the olfactory nerve layer (ONL) and the GL as compared to DMSO controls; suppressed olfactory activity was also reflected by the reduction in tyrosine hydroxylase (TH) staining in PG cells. EPL, External plexiform layer. c, Activity deprivation by dichlobenil reduced the amplitude of sIPSCs in ET cells using whole-cell patch-clamp recordings with a $\mathrm{Cs}^{+}$gluconate internal solution $\left(V_{\mathrm{h}}=0 \mathrm{mV}\right)$. d, Cumulative distributions of sIPSC amplitude in control (black) and deprived (red) conditions ( $\left.n=7\right)$. $\boldsymbol{e}$, Sensory deprivation in the OB reduced levels of GFP in PG cells and synaptic GAD67 surrounding ET cells. White dashed line, Glomeruli; yellow rectangle, magnified region. $f$, Summary of effects by activity deprivation. GFP, $n=170 ; \operatorname{GAD} 67, n=210 .{ }^{* *} p<0.01 ;{ }^{* * *} p<0.001$.

TPMPA block in $G a d 1^{-1-}$ neurons is higher, indicating a presynaptic effect due to less GABA in the synaptic cleft. Although there is some recent evidence for the existence of bicucullineresistant, TPMPA-sensitive synaptic currents in the brain, they are infrequent and have much slower decay (Rosas-Arellano et al., 2011). Our experiments offer the most direct evidence to date documenting a lower transmitter filling at presynaptic terminals of GABAergic neurons lacking GAD67. Intriguingly, quantal amplitude is lower even though it can be influenced by $\mathrm{GABA}_{\mathrm{A}}$ receptor composition as well as the number and location of synapses.

Knockout of GAD67 attenuates, but does not abolish, activitydependent regulation of mIPSCs, suggesting additional mecha- nisms underlying this modulation. For example, other targets of regulation include GAD65 (Hartman et al., 2006), VGAT (De Gois et al., 2005), and postsynaptic $\mathrm{GABA}_{\mathrm{A}}$ receptors (Kilman et al., 2002). Given that only one gene encodes for VGAT, it strongly contributes to GABAergic vesicular filling, as mIPSC amplitude is profoundly depressed in Vgat-null neurons (Wojcik et al., 2006). On the other hand, the low-affinity binding of GABA to VGAT (McIntire et al., 1997) suggests that the cytosolic concentration of GABA could be a critical regulatory checkpoint. Indeed, rapid modulation of GABA reuptake into terminals can be one mechanism that alters its cytosolic concentration (Mathews and Diamond, 2003; Hartmann et al., 2008). Our results corroborate and 
extend previous findings in rat hippocampal neurons (Hartman et al., 2006) that the quantal size of inhibitory transmission can be regulated by changes in vesicle filling, in addition to regulation by postsynaptic receptor composition (Kilman et al., 2002; Saliba et al., 2007; Rannals and Kapur, 2011). For excitatory transmission, a prevailing view is that the postsynaptic sensitivity to glutamate release largely determines the quantal size for an individual synapse, as the vesicular content of glutamate is relatively constant (Lisman et al., 2007). By comparison, inhibitory synapses are distinctive in that they are highly sensitive to the concentration of synaptically released GABA. Several studies indicate that postsynaptic $\mathrm{GABA}_{\mathrm{A}}$ receptors are not saturated (Perrais and Ropert, 2000; Cohen et al., 2000; Rumpel and Behrends, 2000; Hajos et al., 2000; Mathews and Diamond, 2003), thereby allowing fine tuning of quantal transmission via presynaptic changes. We propose that modulation of GAD67 expression and thus GABA production represents a crucial way to equally regulate the inhibitory drive in a network of principal cells.

Emerging evidence suggests that the dysregulated homeostasis that leads to an imbalance of excitation and inhibition could be a common phenomenon among neuropsychiatric disorders such as autism and schizophrenia (Ramocki and Zoghbi, 2008). Interestingly, GAD67 expression is frequently found to be altered in schizophrenic patients (Lewis et al., 2005) and experimental models of schizophrenia (Behrens et al., 2007; Belforte et al., 2010). One hypothesis is that GAD67 is a key target of homeostatic regulation in GABAergic interneurons and that this pathway is involved in schizophrenia (Lisman et al., 2008). In addition to psychiatric disorders, neurological conditions like epilepsy in the hippocampus increase GAD67 expression (Esclapez and Houser, 1999; Ramirez and Gutierrez, 2001), supporting the notion that networks attempt to dampen activity following runaway excitation. These studies suggest that dysregulation of activitydependent compensation involving GAD67 may be an endophenotype present in various neuropsychiatric disorders. Our data provide a plausible explanation for how a failure of homeostasis can lead to altered GAD67 expression and GABAergic function.

In summary, our results unveil a presynaptic form of homeostatic plasticity that is mediated, in part, by regulation of the synthesis and vesicular filling of GABA. Modulation of GAD67 expression and thus GABA production in interneurons may represent a global mechanism to control inhibitory drive in neural circuits.

\section{References}

Asada H, Kawamura Y, Maruyama K, Kume H, Ding RG, Kanbara N, Kuzume H, Sanbo M, Yagi T, Obata K (1997) Cleft palate and decreased brain gamma-aminobutyric acid in mice lacking the $67-\mathrm{kDa}$ isoform of glutamic acid decarboxylase. Proc Natl Acad Sci U S A 94:6496-6499.

Barberis A, Petrini EM, Cherubini E (2004) Presynaptic source of quantal size variability at GABAergic synapses in rat hippocampal neurons in culture. Eur J Neurosci 20:1803-1810.

Behrens MM, Ali SS, Dao DN, Lucero J, Shekhtman G, Quick KL, Dugan LL (2007) Ketamine-induced loss of phenotype of fast-spiking interneurons is mediated by NADPH-oxidase. Science 318:1645-1647.

Belforte JE, Zsiros V, Sklar ER, Jiang Z, Yu G, Li Y, Quinlan EM, Nakazawa K (2010) Postnatal NMDA receptor ablation in corticolimbic interneurons confers schizophrenia-like phenotypes. Nat Neurosci 13:76-83.

Chattopadhyaya B, Di Cristo G, Wu CZ, Knott G, Kuhlman S, Fu Y, Palmiter RD, Huang ZJ (2007) GAD67-mediated GABA synthesis and signaling regulate inhibitory synaptic innervation in the visual cortex. Neuron 54:889-903.

Cohen AS, Lin DD, Coulter DA (2000) Protracted postnatal development of inhibitory synaptic transmission in rat hippocampal area CA1 neurons. J Neurophysiol 84:2465-2476.
Davis GW, Bezprozvanny I (2001) Maintaining the stability of neural function: a homeostatic hypothesis. Annu Rev Physiol 63:847-869.

De Gois S, Schäfer MK, Defamie N, Chen C, Ricci A, Weihe E, Varoqui H, Erickson JD (2005) Homeostatic scaling of vesicular glutamate and GABA transporter expression in rat neocortical circuits. J Neurosci 25:7121-7133.

Esclapez M, Houser CR (1999) Up-regulation of GAD65 and GAD67 in remaining hippocampal GABA neurons in a model of temporal lobe epilepsy. J Comp Neurol 412:488-505.

Esclapez M, Tillakaratne NJ, Kaufman DL, Tobin AJ, Houser CR (1994) Comparative localization of two forms of glutamic acid decarboxylase and their mRNAs in rat brain supports the concept of functional differences between the forms. J Neurosci 14:1834-1855.

Fenalti G, Law RH, Buckle AM, Langendorf C, Tuck K, Rosado CJ, Faux NG, Mahmood K, Hampe CS, Banga JP, Wilce M, Schmidberger J, Rossjohn J, El-Kabbani O, Pike RN, Smith AI, Mackay IR, Rowley MJ, Whisstock JC (2007) GABA production by glutamic acid decarboxylase is regulated by a dynamic catalytic loop. Nat Struct Mol Biol 14:280-286.

Gogolla N, Galimberti I, DePaola V, Caroni P (2006) Preparation of organotypic hippocampal slice cultures for long-term live imaging. Nat Protoc 1:1165-1171.

Hájos N, Nusser Z, Rancz EA, Freund TF, Mody I (2000) Cell type- and synapse-specific variability in synaptic GABAA receptor occupancy. Eur J Neurosci 12:810-818.

Hartman KN, Pal SK, Burrone J, Murthy VN (2006) Activity-dependent regulation of inhibitory synaptic transmission in hippocampal neurons. Nat Neurosci 9:642-649.

Hartmann K, Bruehl C, Golovko T, Draguhn A (2008) Fast homeostatic plasticity of inhibition via activity-dependent vesicular filling. PLoS One 3:e2979.

Henneberger C, Kirischuk S, Grantyn R (2005) Brain-derived neurotrophic factor modulates GABAergic synaptic transmission by enhancing presynaptic glutamic acid decarboxylase 65 levels, promoting asynchronous release and reducing the number of activated postsynaptic receptors. Neuroscience 135:749-763.

Hensch TK, Fagiolini M, Mataga N, Stryker MP, Baekkeskov S, Kash SF (1998) Local GABA circuit control of experience-dependent plasticity in developing visual cortex. Science 282:1504-1508.

Huang ZJ, Di Cristo G, Ango F (2007) Development of GABA innervation in the cerebral and cerebellar cortices. Nat Rev Neurosci 8:673-686.

Kash SF, Johnson RS, Tecott LH, Noebels JL, Mayfield RD, Hanahan D, Baekkeskov S (1997) Epilepsy in mice deficient in the 65-kDa isoform of glutamic acid decarboxylase. Proc Natl Acad Sci U S A 94:14060-14065.

Kilman V, van Rossum MC, Turrigiano GG (2002) Activity deprivation reduces miniature IPSC amplitude by decreasing the number of postsynaptic GABA(A) receptors clustered at neocortical synapses. J Neurosci 22:1328-1337.

Kubo K, Nishikawa K, Hardy-Yamada M, Ishizeki J, Yanagawa Y, Saito S (2009) Altered responses to propofol, but not ketamine, in mice deficient in the 65-kilodalton isoform of glutamate decarboxylase. J Pharmacol Exp Ther 329:592-599.

Kundakovic M, Chen Y, Costa E, Grayson DR (2007) DNA methyltransferase inhibitors coordinately induce expression of the human reelin and glutamic acid decarboxylase 67 genes. Mol Pharmacol 71:644-653.

Lewis DA, Hashimoto T, Volk DW (2005) Cortical inhibitory neurons and schizophrenia. Nat Rev Neurosci 6:312-324.

Liang F, Isackson PJ, Jones EG (1996) Stimulus-dependent, reciprocal upand downregulation of glutamic acid decarboxylase and $\mathrm{Ca}^{2+}$ / calmodulin-dependent protein kinase II gene expression in rat cerebral cortex. Exp Brain Res 110:163-174.

Liang SL, Carlson GC, Coulter DA (2006) Dynamic regulation of synaptic GABA release by the glutamate-glutamine cycle in hippocampal area CA1. J Neurosci 26:8537-8548.

Lisman JE, Raghavachari S, Tsien RW (2007) The sequence of events that underlie quantal transmission at central glutamatergic synapses. Nat Rev Neurosci 8:597-609.

Lisman JE, Coyle JT, Green RW, Javitt DC, Benes FM, Heckers S, Grace AA (2008) Circuit-based framework for understanding neurotransmitter and risk gene interactions in schizophrenia. Trends Neurosci 31:234-242.

Mathews GC, Diamond JS (2003) Neuronal glutamate uptake Contributes to GABA synthesis and inhibitory synaptic strength. J Neurosci 23:2040-2048. 
McIntire SL, Reimer RJ, Schuske K, Edwards RH, Jorgensen EM (1997) Identification and characterization of the vesicular GABA transporter. Nature 389:870-876.

Obata K, Hirono M, Kume N, Kawaguchi Y, Itohara S, Yanagawa Y (2008) GABA and synaptic inhibition of mouse cerebellum lacking glutamate decarboxylase 67. Biochem Biophys Res Commun 370:429-433.

Parrish-Aungst S, Kiyokage E, Szabo G, Yanagawa Y, Shipley MT, Puche AC (2011) Sensory experience selectively regulates transmitter synthesis enzymes in interglomerular circuits. Brain Res 1382:70-76.

Patz S, Wirth MJ, Gorba T, Klostermann O, Wahle P (2003) Neuronal activity and neurotrophic factors regulate GAD-65/67 mRNA and protein expression in organotypic cultures of rat visual cortex. Eur J Neurosci 18:1-12.

Perrais D, Ropert N (2000) Altering the concentration of GABA in the synaptic cleft potentiates miniature IPSCs in rat occipital cortex. Eur J Neurosci 12:400-404.

Ramírez M, Gutiérrez R (2001) Activity-dependent expression of GAD67 in the granule cells of the rat hippocampus. Brain Res 917:139-146.

Ramocki MB, Zoghbi HY (2008) Failure of neuronal homeostasis results in common neuropsychiatric phenotypes. Nature 455:912-918.

Rannals MD, Kapur J (2011) Homeostatic strengthening of inhibitory synapses is mediated by the accumulation of $\mathrm{GABA}_{\mathrm{A}}$ receptors. J Neurosci 31:17701-17712.

Rosas-Arellano A, Parodi J, Machuca-Parra AI, Sánchez-Gutiérrez A, Inestrosa NC, Miledi R, Martínez-Torres A (2011) The GABA(A) $\rho$ receptors in hippocampal spontaneous activity and their distribution in hippocampus, amygdala and visual cortex. Neurosci Lett 500:20-25.

Rosato-Siri M, Grandolfo M, Ballerini L (2002) Activity-dependent modulation of GABAergic synapses in developing rat spinal networks in vitro. Eur J Neurosci 16:2123-2135.

Rumpel E, Behrends JC (2000) Postsynaptic receptor occupancy during evoked transmission at striatal GABAergic synapses in vitro. J Neurophysiol 84:771-779.

Rutherford LC, DeWan A, Lauer HM, Turrigiano GG (1997) Brain-derived neurotrophic factor mediates the activity-dependent regulation of inhibition in neocortical cultures. J Neurosci 17:4527-4535.

Saliba RS, Michels G, Jacob TC, Pangalos MN, Moss SJ (2007) Activitydependent ubiquitination of $\mathrm{GABA}_{\mathrm{A}}$ receptors regulates their accumulation at synaptic sites. J Neurosci 27:13341-13351.

Shao Z, Puche AC, Kiyokage E, Szabo G, Shipley MT (2009) Two GABAergic intraglomerular circuits differentially regulate tonic and phasic presynaptic inhibition of olfactory nerve terminals. J Neurophysiol 101:1988-2001.
Soghomonian JJ, Martin DL (1998) Two isoforms of glutamate decarboxylase: why? Trends Pharmacol Sci 19:500-505.

Song I, Savtchenko L, Semyanov A (2011) Tonic excitation or inhibition is set by $\mathrm{GABA}(\mathrm{A})$ conductance in hippocampal interneurons. Nat Commun 2:376.

Stellwagen D, Malenka RC (2006) Synaptic scaling mediated by glial TNFalpha. Nature 440:1054-1059.

Stork O, Ji FY, Kaneko K, Stork S, Yoshinobu Y, Moriya T, Shibata S, Obata K (2000) Postnatal development of a GABA deficit and disturbance of neural functions in mice lacking GAD65. Brain Res 865:45-58.

Suzuki N, Bekkers JM (2010) Inhibitory neurons in the anterior piriform cortex of the mouse: classification using molecular markers. J Comp Neurol 518:1670-1687.

Swanwick CC, Murthy NR, Kapur J (2006) Activity-dependent scaling of GABAergic synapse strength is regulated by brain-derived neurotrophic factor. Mol Cell Neurosci 31:481-492.

Tamamaki N, Yanagawa Y, Tomioka R, Miyazaki J, Obata K, Kaneko T (2003) Green fluorescent protein expression and colocalization with calretinin, parvalbumin, and somatostatin in the GAD67-GFP knock-in mouse. J Comp Neurol 467:60-79.

Tian N, Petersen C, Kash S, Baekkeskov S, Copenhagen D, Nicoll R (1999) The role of the synthetic enzyme GAD65 in the control of neuronal gamma-aminobutyric acid release. Proc Natl Acad Sci U S A 96:12911-12916.

Tremolizzo L, Carboni G, Ruzicka WB, Mitchell CP, Sugaya I, Tueting P, Sharma R, Grayson DR, Costa E, Guidotti A (2002) An epigenetic mouse model for molecular and behavioral neuropathologies related to schizophrenia vulnerability. Proc Natl Acad Sci U S A 99:17095-17100.

Turrigiano GG, Nelson SB (2004) Homeostatic plasticity in the developing nervous system. Nat Rev Neurosci 5:97-107.

Tyler WJ, Petzold GC, Pal SK, Murthy VN (2007) Experience-dependent modification of primary sensory synapses in the mammalian olfactory bulb. J Neurosci 27:9427-9438.

Wilson RI, Mainen ZF (2006) Early events in olfactory processing. Annu Rev Neurosci 29:163-201.

Wojcik SM, Katsurabayashi S, Guillemin I, Friauf E, Rosenmund C, Brose N, Rhee JS (2006) A shared vesicular carrier allows synaptic corelease of GABA and glycine. Neuron 50:575-587.

Yanagawa Y, Kobayashi T, Kamei T, Ishii K, Nishijima M, Takaku A, Tamura S (1997) Structure and alternative promoters of the mouse glutamic acid decarboxylase 67 gene. Biochem J 326:573-578.

Yoon H, Enquist LW, Dulac C (2005) Olfactory inputs to hypothalamic neurons controlling reproduction and fertility. Cell 123:669-682. 